\title{
Delays in diagnosis and treatment among children with cancer: Egyptian perspective
}

Suzy Abdelmabood ', Shaimaa Kandil' ${ }^{2}$, Ahmed Megahed $^{2}$, AshrafFouda $^{1}$

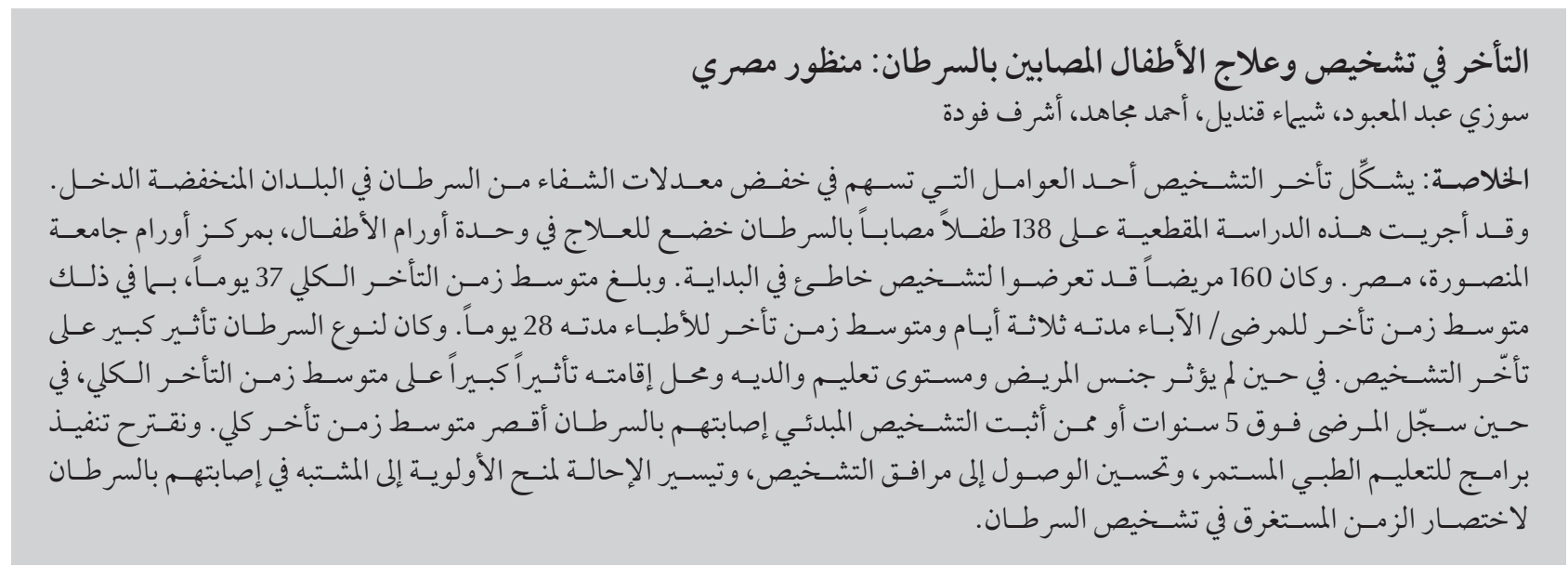

ABSTRACT Delayed diagnosis is one of the contributing factors to lower cure rates for cancer in low-income countries. This was a cross-sectional study of 138 children with cancer who were treated at the Pediatric Oncology Unit, Oncology Center of Mansoura University, Egypt. One hundred and sixteen patients were initially misdiagnosed. The median total delay was 37 days, including median patient/parent delay of 3 days and median physician delay of 28 days. The type of cancer significantly influenced the diagnostic delay. Patients' sex, level of parents' education, and residence did not significantly affect the median total delay, while patients aged < 5 years and those who had an initial provisional diagnosis of cancer had the shortest median total delay. We suggest implementation of continuing medical education programmes, improving access to diagnostic facilities, and facilitating referral to give priority to those with suspected cancer to shorten the time for cancer diagnosis.

\section{Délais de diagnostic et de traitement chez les enfants atteints de cancer : perspective égyptienne}

RÉSUMÉ Les délais de diagnostic constituent l'un des facteurs contribuant à des taux de guérison moins élevés du cancer dans les pays à revenu faible. La présente étude transversale a été menée auprès de 138 enfants atteints de cancer et traités dans I'Unité d'oncologie pédiatrique du Centre d'oncologie de l'Université de Mansoura, en Égypte. Cent-seize patients avaient été mal diagnostiqués à l'origine. Le délai total médian était de 37 jours, avec un délai patient/parent médian de trois jours et un délai médecin médian de 28 jours. Le type de cancer avait une influence significative sur le délai de diagnostic. Le sexe du patient, le niveau d'éducation des parents et le lieu de résidence n'affectaient pas significativement le délai total médian. En revanche, les patients de moins de cinq ans et ceux avec un diagnostic de cancer provisoire posé initialement bénéficiaient du délai total médian le plus court. Nous suggérons de mettre en place des programmes de formation médicale continue, d'améliorer l'accès aux services de diagnostic, et de faciliter l'orientation-recours de façon à donner la priorité aux patients suspects de cancer et ainsi raccourcir le délai de diagnostic.

${ }^{7}$ Hematology/Oncology Unit (Correspondence to: A. Fouda: ashraf_foda@mans.edu.eg) 2Pediatric Department, Mansoura University Children's Hospital, Faculty of Medicine, Mansoura University, Mansoura, Egypt.

Received: 29/12/15; accepted: 06/11/16 


\section{Introduction}

Overall, cancer remains a rare diagnosis in children. In developed countries, childhood cancer represents $<1 \%$ of all cases of cancer; however, in lowresource or developing countries, where children may make up half the population, the proportion of childhood cancer can be 5 times higher in comparison. Cancer remains an important cause of childhood mortality, with an estimated 80000 cancer-related deaths per year worldwide. Although overall survival rates for childhood cancer are $\sim 80 \%$ in high-income countries, they may be as low as $10 \%$ in developing countries. This may be due to the lack of proper diagnosis, delayed detection and slow referral for treatment (1).

Diagnosis of childhood cancer as early as possible is crucial to reduce mortality. Children with cancer have improved response to treatment compared to those who are older, but childhood cancer progresses faster in the absence of treatment than in older individuals (2). High cure rate in cancer needs right diagnosis, quick referral to medical care system, and finishing the required treatment. Cure rates are at least 30\% lower in low-income than higher-income countries (3), which may be due to delay in diagnosis (advanced stage of cancer), more fatal toxicities, incomplete or abandonment of treatment (4).

Few studies have been published on determinants and impacts of diagnostic delay in childhood cancer. Practical new strategies to reduce diagnostic delay are needed and require study and understanding of diagnostic delays and their impact on the prognosis of cancer (5). To the best of our knowledge, few studies have been conducted in Egypt to assess diagnostic delay in childhood cancer. We evaluated different factors resulting in diagnostic delay in 138 children aged 1 month to 17 years, who were diagnosed with cancer or referred to the Pediatric Oncology Unit,
Oncology Center of Mansoura University, Egypt. Our Oncology Center is a regional tertiary referral centre for children with cancer in the Egyptian Delta Region, which has $~ 5.5$ million inhabitants.

\section{Methods}

\section{Setting}

This was a cross-sectional study of children diagnosed with cancer at our institution from November 2013 to January 2015. Of the 168 patients approached, 138 agreed to participate. There were 77 boys (55.8\%) and 61 girls (44.2\%), with a male to female ratio of 1.0: 0.8 and age range of 1 month to 17 years.

\section{Data collection}

Data were collected from parent interviews upon admission to the Pediatric Oncology Unit. All referral documents (letters, imaging and laboratory study reports) were reviewed and examined to establish approximate dates of initial medical contact, diagnostic tests and initial diagnosis. We estimated the time in days from the onset of patients' symptoms to the beginning of cancer treatment (total delay). Different components of delay were obtained from the date of onset of symptoms until the first medical contact (patient/parent delay); time from initial medical contact until the patient was assessed by an oncologist (referral delay); time from referral to an oncologist until the final diagnosis of cancer (diagnostic delay); and time from diagnosis of cancer until the start of treatment (treatment delay) (Figure 1). The term misdiagnosis was used when another benign disease was diagnosed provisionally and cancer was not suspected.

The patients' characteristics included: age at diagnosis, sex, parental educational level, residence, duration of illness at diagnosis, type of malignancy, initial symptoms, initial diagnosis by a healthcare professional, and initial physicians' notes before the final diagnosis of malignancy was recorded. The most common malignancies were: leukaemia (including acute lymphoblastic leukaemia and acute myeloid leukaemia), lymphoma [Hodgkin's disease (HD) and non-Hodgkin's lymphoma (NHL)], Wilms' tumour, neuroblastoma (NB), rhabdomyosarcoma (RMS), bone tumours (Ewing sarcoma and osteosarcoma), germ cell tumours (GCTs), brain tumours, and histiocyte disorders.

\section{Statistical analysis}

Data were analysed using SPSS version 21.0 (Chicago, IL, USA). Numerical data were presented as mean (standard deviation; SD) if normally distributed and as median and interquartile range (IQR) (25th-75th centiles) if not normally distributed. Delay times were mostly right-skewed, so nonparametric tests were used. The Mann-Whitney $\mathrm{U}$ test was used to compare between 2 groups and the Kruskal-Wallis test to compare among $>2$ groups. Categorical data were compared using the $\chi^{2}$ test. $P<0.05$ was considered to indicate statistical significance.

\section{Results}

Sex, age at diagnosis, level of parents' education, residence, initial provisional suspicion of malignancy and type of malignancy, and their relationship to the total delay in cancer diagnosis are shown in Table 1. Sex and level of parents' education did not significantly affect median total delay. In contrast, age at diagnosis had a significant effect on total delay, as patients aged $<5$ years at diagnosis had the shortest median total delay. When malignancy was initially suspected, the median total delay for these patients was significantly shorter. The median total delay was significantly influenced by the type of cancer; the shortest delay was observed among 


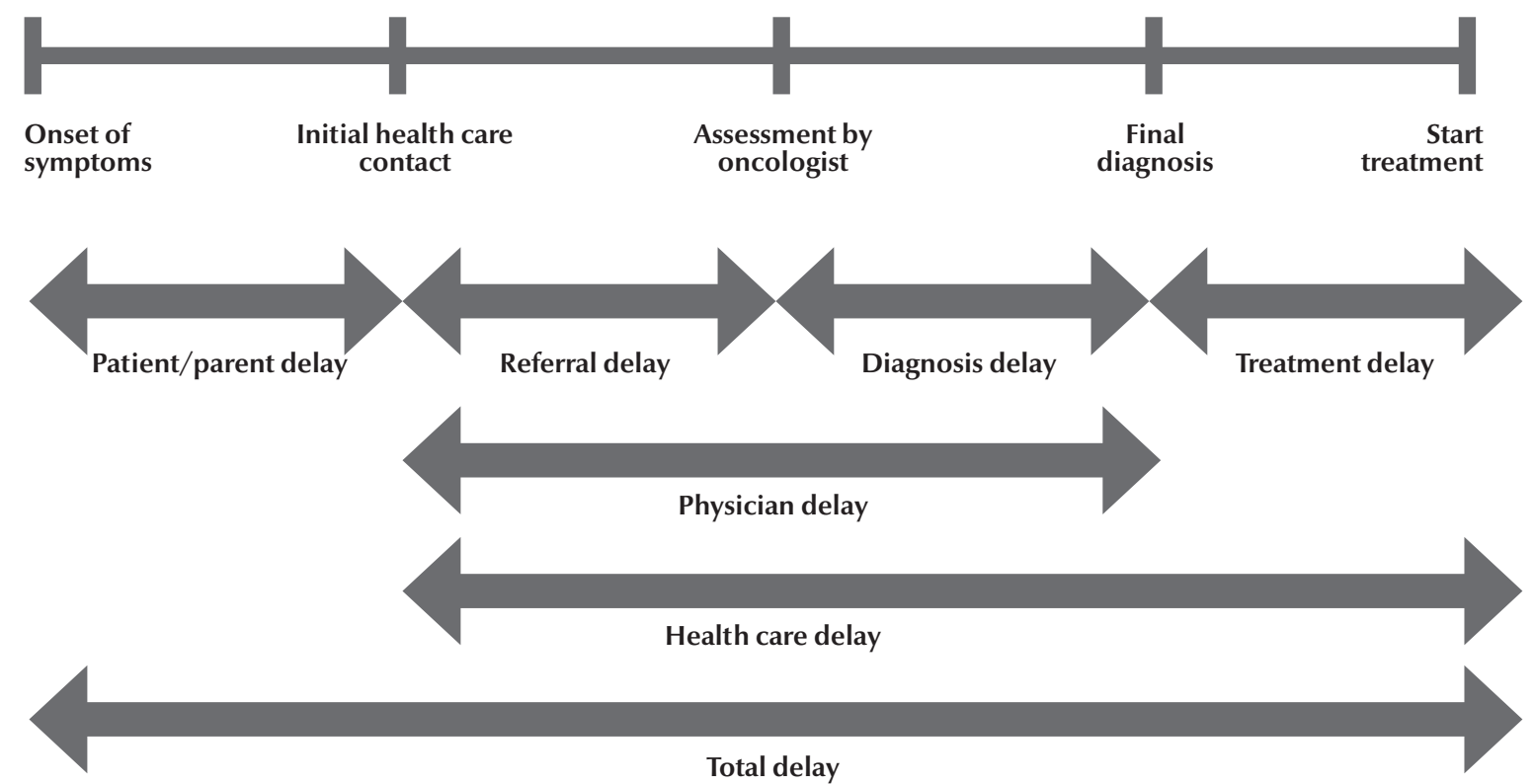

patients with Wilms' tumour, followed by acute leukaemia and NHL.

Most of the patients were diagnosed at an advanced stage of malignancy as follows: NHL: 1, 14 and 7 patients had stage I, III and IV, respectively; NB: 3, 4 and 8 patients had stage II, III and IV, respectively; HD: 1, 1, 2 and 1 patient had stage I, II, III and IV, respectively); Wilms' tumour: 2, 3, 4 and 1 patient had stage I, II, III and IV, respectively); bone tumours (Ewing sarcoma and osteosarcoma): 2, 6, 2 and 2 patients had stage T1N0M0, T2N0M0, T2N1M1 and T3N1M0, respectively.

Median total delay for all patients was 37.0 days (IQR 21.5-67.0 days, range 1.0-417.0 days). Median patient/ parent diagnostic delay was 3.0 days (IQR 1.0-7.0 days, range 1.0-365.0 days). The shortest delay was in patients with Wilms' tumour, hepatoblastoma, Langerhans cell histocytosis ( $\mathrm{LCH}$ ), leukaemia and NB. The longest delay was 101.0 days in patients with HD. Median physician delay (referral + diagnostic delay) was 28.0 days (IQR $15.0-51.0$ days, range 1.0-312.0 days). The shortest delay was in patients with GCT, NHL, brain tumours and leukaemia. Median referral delay was 14.5 days (IQR 3.0-30.0 days, range 1.0-300.0 days). The shortest delay was in patients with GCT, Wilms' tumour, NHL and leukaemia. Median diagnostic delay was 12.0 days (IQR 8.0-17.25 days, range 1.0-120.0 days). The shortest delay was in patients with hepatoblastoma, leukaemia, brain tumours and RMS. Median treatment delay was 1.0 day (IQR 0-1.0 day, range 1.0-60.0 days). The median treatment delay was $1.0-2.5$ days for different types of tumours. Median health system delay (physician + treatment delays) was 30.0 days (IQR 16.0-54.0 days, range 7.0-313.0 days). The shortest duration was in patients with GCT, Wilms' tumour, NHL and leukaemia. Different components of delay lengths for each type of cancer: patient/parent delay, referral delay, diagnostic delay, physician delay, treatment delay and health system delay are shown with the mean age at the start of symptoms (Table 2).

Common initial presenting symptoms for our patients were: fever, which was the most common initial symptom in 44 patients, followed by bone aches, limping or difficulty walking in 26 cases, abdominal symptoms (pain, enlargement and mass) in 20 patients and pallor in 16 patients (Table 3 ).

Diagnostic clues suggestive for initial provisional diagnosis of cancer (according to different types of cancer) were: complete blood count, which was helpful for suspecting malignancy in $41.3 \%$ of cases; imaging such as abdominal ultrasound, X ray, computed tomography and magnetic resonance imaging was suggestive of cancer in 29.7, 9.4, 2.9 and $8.0 \%$ of cases, respectively (Table 3). Biopsies were essential to differentiate malignancy from other benign conditions in $8.7 \%$ of patients.

In an attempt for physicians to reach a diagnosis or explain their patients' complaints, provisional initial diagnoses ranged from everyday minor problems (e.g., common cold/pharyngitis and tonsillitis) to more serious diagnoses such as arthritis, myositis, osteomyelitis or juvenile rheumatoid arthritis to explain symptoms like fever and feeling unwell, bone pain and limb swelling. Pallor and anaemia were interpreted as iron deficiency anaemia (14 patients) or favism (1 patient). Abdominal 


\begin{tabular}{|c|c|c|c|}
\hline Patients characteristics & No. of patients (\%) & $\begin{array}{c}\text { Total delay (d) } \\
\text { Median (IQR: 25th-75th) }\end{array}$ & $\boldsymbol{P}$ \\
\hline \multicolumn{4}{|l|}{ Sex } \\
\hline Male & $77(55.8 \%)$ & $29.0(19.0-48.0)$ & \multirow[t]{2}{*}{0.590} \\
\hline Female & $61(44.2 \%)$ & $42.0(25.0-76.5)$ & \\
\hline \multicolumn{4}{|l|}{ Age } \\
\hline $0-5$ years & $77(55.8 \%)$ & $29.0(19.0-50.5)$ & \multirow[t]{3}{*}{$0.038^{*}$} \\
\hline $5-10$ years & $28(20.3 \%)$ & $39.0(25.5-66.5)$ & \\
\hline$>10$ years & $33(23.9 \%)$ & $45.0(27.5-145.5)$ & \\
\hline \multicolumn{4}{|l|}{ Parents level of education } \\
\hline No education (illiterate) & $22(15.9 \%)$ & $39.50(26.75-64.5)$ & \multirow[t]{3}{*}{0.727} \\
\hline Low education (below high school) & $98(71.0 \%)$ & $35.50(20.0-70.25)$ & \\
\hline High education & $18(13 \%)$ & $33.0(15.5-75.5)$ & \\
\hline \multicolumn{4}{|l|}{ Patient residence } \\
\hline Urban area & $63(45.7 \%)$ & $32(19.0-64.0)$ & \multirow[t]{2}{*}{0.564} \\
\hline Rural area & $75(54.3 \%)$ & $39(22.0-71.0)$ & \\
\hline \multicolumn{4}{|l|}{ Initial provisional diagnosis } \\
\hline Correct (suspected cancer) & $22(15.9 \%)$ & $19.50(12.75-39.0)$ & \multirow[t]{2}{*}{$0.039 *$} \\
\hline Wrong diagnosis & $116(84.1 \%)$ & $38.0(24.25-70.45)$ & \\
\hline \multicolumn{4}{|l|}{ Type of malignancy } \\
\hline Leukaemia (ALL+AML) & $64(45.7 \%)$ & $31.0(19.0-46.5)$ & \multirow[t]{12}{*}{$0.026^{*}$} \\
\hline Hodgkin's disease & $5(3.5 \%)$ & $240.0(44.5-368.5)$ & \\
\hline Non-Hodgkin's lymphoma & $22(15.9 \%)$ & $32.0(23.75-55.25)$ & \\
\hline Neuroblastoma & $15(10.5 \%)$ & $39.0(28.0-70.0)$ & \\
\hline Wilms' tumour & $10(7.0 \%)$ & $22.50(13.5-32.5)$ & \\
\hline Rhabdomyosarcoma & $2(1.4 \%)$ & $88.5(40.0-137.0)$ & \\
\hline Ewing sarcoma & $6(4.4 \%)$ & $72.50(60.75-173.25)$ & \\
\hline Osteosarcoma & $6(4.4 \%)$ & $130.50(62.0-205.25)$ & \\
\hline GCT & $3(2.2 \%)$ & $12.0(1.0-15.0)$ & \\
\hline $\mathrm{LCH}$ & $2(1.4 \%)$ & $39.50(24.0-55.0)$ & \\
\hline Brain tumour & $2(1.4 \%)$ & $44.0(7.0-81.0)$ & \\
\hline Hepatocellular carcinoma & $1(0.7 \%)$ & 187.0 & \\
\hline
\end{tabular}

*Significant at $P<0.05$ (Mann-Whitney $U$ test to compare between two groups, and Kruskal-Wallis to compare among $>2$ groups). $A L L=$ acute lymphoblastic leukaemia; $A M L$ : acute myeloid leukaemia; $G C T=$ germ cell tumour; $L C H=$ Langerhans cells histocytosis.

symptoms were misdiagnosed as gastroenteritis/gastritis, parasitic infestation or even hepatitis (Table 3).

Initial healthcare providers contacted were paediatricians $(47.1 \%$ of patients), general practitioners ( $13.8 \%$ of patients) and internal medicine physicians ( $15.9 \%$ of patients). Some patients initially contacted physicians in other specialties due to different disease presentations: for example, orthopaedics (for arthralgia, arthritis or bone pain) or ophthalmology (for proptosis or raccoon eyes). There was no significant difference in physician diagnostic delay regardless of whether the first physician contacted was a paediatrician $(P=0.959)$. One hundred and sixteen (84.1\%) patients were initially wrongly diagnosed (cancer was not suspected), and 22 patients (15.9\%) were provisionally suspected of having cancer from the start of seeking medical advice (Table 3). Malignancy was correctly suspected in 10 of 64 patients with leukaemia, 6 of 10 with Wilms' tumour, 3 of 22 with NHL, 2 of 3 with GCT, and 1 of 15 with NB. None of the patients with bone tumours (12 patients), HD (5 patients), RMS (2 patients), brain tumors (2 patients), LCH (2 patients) or hepatoblastoma (1 patient) were correctly suspected to have malignancy. Most of the patients (13 of 22; 59.1\%) correctly suspected for malignancy, were first seen by a paediatrician. 


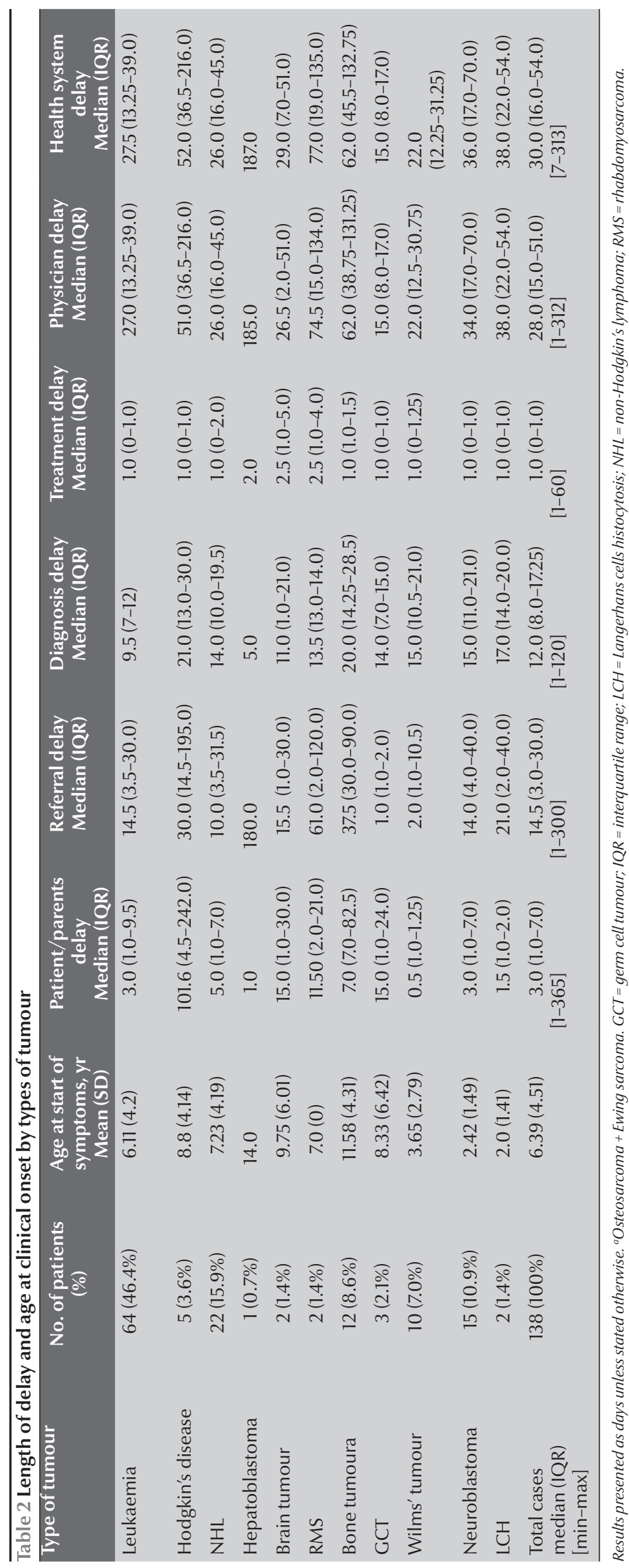

\section{Discussion}

Although the outcomes of childhood cancer are better to some extent than those of adult cancer, the tumours grow at a faster rate unless they are treated. This can be partially overcome by early diagnosis (2). In this study, the median total delay was 37 days, which was shorter than that reported by a recent study from Egypt (47 days) (6). However, our delay was longer than in previous Canadian (7) and South African (2) studies that reported a median total diagnostic delay of 30 and 34 days, respectively. Differences in healthcare systems, physician attitudes and concerns about childhood cancer, and socioeconomic and cultural factors may have been responsible for the different results.

The median patient/parent delay was 3 days, which is shorter than in previous Egyptian (6), Canadian (7) and South African (2) studies (8, 9 and 5 days, respectively). This may have been because $55.8 \%$ of the study population that had the shortest parent delay was aged $<5$ years.

The median physician delay was 28 days, which was longer than in previous studies from Canada, South Africa and Turkey ( 8,20 and 23 days, respectively) $(2,7,8)$. This may have been due to difficulties facing the physicians or lack of awareness of presentation and diagnosis of childhood cancer. It might also be explained by the presence of other major concerns for the physicians in developing countries, like malnutrition, infectious disease and parasitic infections rather than suspecting malignancies in children. It may also have been due to lack of facilities and equipment for diagnosis of cancer in developing countries.

Sex of the patients did not significantly affect the time to diagnosis. This agrees with previous studies (2,6-8), although other studies (9-11) have 


\begin{tabular}{|c|c|}
\hline Different clinical and diagnostic parameters & $\begin{array}{c}\text { Total no. of } \\
\text { patients } \\
(\%)\end{array}$ \\
\hline \multicolumn{2}{|l|}{ Main initial presenting symptoms } \\
\hline Fever & 44 \\
\hline Bone pain/limping/difficulty walking & 26 \\
\hline Abdominal pain/enlargement/mass & 20 \\
\hline Pallor & 16 \\
\hline Lymph node swelling & 15 \\
\hline Constipation/diarrhoea, vomiting & 8 \\
\hline Swelling (jaw, skull or legs) & 7 \\
\hline Bleeding (hematemesis/melena, vaginal bleeding, epistaxis) & 5 \\
\hline Cough/respiratory distress & 4 \\
\hline $\begin{array}{l}\text { Rare: testicular swelling, eye (proptosis), jaundice, } \\
\text { priapism, polyphagia/polysomnia }\end{array}$ & $3,2,1,1,1$ \\
\hline \multicolumn{2}{|l|}{ Diagnostic clues suggestive of cancer diagnosis } \\
\hline Complete blood count & $57(41.3 \%)$ \\
\hline $\mathrm{X}$ ray & $13(9.4 \%)$ \\
\hline Abdominal ultrasonography & $41(29.7 \%)$ \\
\hline Computed tomography & $4(2.9 \%)$ \\
\hline Magnetic resonance imaging & $11(8.0 \%)$ \\
\hline Biopsy & $12(8.7 \%)$ \\
\hline Misdiagnosis initially (cancer was not suspected) & $116(84.1 \%)$ \\
\hline Common cold/pharyngitis/tonsillitis & 17 \\
\hline Arthritis/myositis & 15 \\
\hline Anaemia (iron deficiency/favism) & 15 \\
\hline Infections (UTI, typhoid, appendicitis, osteomyelitis, septicaemia) & 14 \\
\hline Gastroenteritis/gastritis & 7 \\
\hline Parasitic infestation (worms) & 7 \\
\hline Lymphadenitis & 6 \\
\hline Bronchitis/bronchial asthma & 4 \\
\hline Radiculitis/disc prolapse/GBS/post-injection injury & 4 \\
\hline Hepatitis & 3 \\
\hline Bleeding tendency/normal menarche & 3 \\
\hline Functional constipation/food poisoning & 2 \\
\hline Correctly suspected (malignancy suspected initially) & $22(15.9 \%)$ \\
\hline
\end{tabular}

GBS = Guillain-Barré syndrome; UTI = urinary tract infection .

reported a significantly longer delay for male patients.

Patients aged $<5$ years had the shortest diagnostic delay, which was similar to a previous systematic review that showed that time to diagnosis was significantly shorter for younger children (12). Probable interpretations are slow-growing tumours (ganglioglioma or localized Ewing sarcoma) are more common in older children in comparison to some rapid-growing aggressive tumours (nephroblastoma, leukaemia or choroid plexus carcinoma), which are common in younger age groups (12).

Unexpectedly, the level of parents' education did not significantly influence the diagnostic delay. This can be explained by the small number of parents with higher educational levels in our study $(n=18 ; 13 \%)$, which was similar to a South African study with a low numbers of highly educated parents, which did not affect the delay significantly (2). These results differ from a Canadian study reporting a greater percentage of highly educated parents (41.8\%) who had a significantly shorter median total delay (6).

Whether the patients were from a rural or urban area did not significantly affect total delay time. This may have been due to the small surface area of the Delta Region that is covered by our hospital, as the patients can reach most healthcare facilities (university hospitals, regional local hospitals or private clinics) within a short period of time. This finding was similar to another study from the Egyptian Delta (6).

The diagnostic delay was significantly influenced by the type of tumour. The shortest delay was observed for Wilms' tumour (median 22.5 days) followed by acute leukaemia (31 days) and NHL (32 days). This may have been due to the younger age at the time of symptom onset among Wilms' tumour patients and the large abdominal mass in relation to small body size making the tumour easily recognizable. Our results were consistent with most of the previous studies that found shorter diagnostic delay for leukaemia, renal tumours, NB and NHL; intermediate delay for HD, RMS, GCT and retinoblastoma; and longer delay for brain tumours, bone tumours and soft-tissue sarcoma $(5,7,12,13)$. This also may be 
explained by different tumour growth patterns: slowly growing patterns tumours, such as low-grade astrocytoma, ganglioglioma or localized Ewing sarcoma are diagnosed more slowly, whereas aggressive fast-growing tumours such as Wilms' tumour, leukaemia and NHL are diagnosed more quickly (12). The median total delay for leukaemia was 31 days, which was the same as in a South African study (2), while the median diagnosis delay in a Canadian study was 18 days (7). The shortest median physician delay for leukaemia was in Canada (3 days) (7), while it was 27 days in the current study and 22 days in the South African study (2). Reasons for this large difference could be that the physicians may have had difficulty suspecting and recognizing symptoms of leukaemia, or unavailability of diagnostic facilities such as bone marrow examination and flow cytometry.

The longest total delay in the current study was for diagnosis of $\mathrm{HD}$ and bone tumours. This may have been partly related to the quiescent nature of $\mathrm{HD}$, making the patient/parent delay long (median 101 days). Also, bone tumour diagnosis was characterized by long physician delay, which may have been because of the slow growing nature of these tumours, wrong initial diagnoses and lack of facilities for diagnosis (imaging or open bone biopsy).

Eighty-four percent of our patients were initially misdiagnosed and only $16 \%$ were suspected to have cancer at the time of their first visit to a physician. More than half of those suspected to have cancers initially (59.1\%) were seen by a paediatrician, which indicated the awareness of paediatricians (to some extent) for cancer occurrence in children.
Most of the correctly suspected patients had leukaemia and Wilms' tumour. Leukaemia was suspected because of prolonged fever, pallor, bleeding tendency and abdominal enlargement. Wilms' tumour was the only tumour with a higher percentage of initial correct diagnoses compared to other tumours, which may be for the reasons mentioned before. This differs from another study from Egypt that reported that misdiagnosis was initially recorded in $39.5 \%$ of patients (6), but was similar to previous studies (13-15) showing that diagnostic delay was significantly longer when patients were seen for the first time by a general practitioner rather than a paediatrician.

Common presentations of cancer in our study were fever, bone pain, difficulty walking and abdominal enlargement/pain, which denote that previous such complaints may be serious. With presentation of fever, especially when it is prolonged or of unknown origin, malignancy should be included in the differential diagnosis, which requires thorough clinical examination and further investigation. Abdominal pain, although it is a common paediatric problem, may signify serious illness, especially when it is persistent, interfering with daily activities or accompanied by abdominal enlargement, which may necessitate abdominal ultrasonography.

Our study had several limitations. First, the small sample size and that most of the patients were from the Egyptian Delta Region. Second, recall bias was an issue when questioning about the beginning of symptoms, but we attempted to achieve accuracy by reviewing dates of referral letters and initial written suspected diagnoses. Third, there was difficulty in evaluating the socioeconomic status of parents due to cultural issues, as most of the parents refused to give information when they were asked about their income.

In conclusion, we suggest shortening diagnostic delay through the implementation of continuing medical education programmes for healthcare providers, which is not obligatory yet in Egypt, and specialty medical training about childhood cancer and the mode of cancer presentation. Parents should be encouraged to seek medical advice from specialists, such as paediatricians, to help with early recognition of alarm signs. This will improve the diagnostic skills of the physicians, their index of suspicion, and facilitate and enhance the healthcare system to minimize the time to referral for a child with possible cancer. Also, facilitating referral to give priority to those with suspected cancer diagnosis, while improving access to diagnostic facilities, will help to shorten the time to final diagnosis of cancer.

\section{Acknowledgements}

We would like to thank Dr. Lesleigh Abbott, MD, FRCPC, Assistant Professor, Hematologist/Oncologist, The Children's Hospital of Eastern Ontario (CHEO), Ottawa, Ontario, Canada, for English language review and correction of the manuscript. We would also like to thank Dr. Soha Aly Elmorsy, MD, PhD, Research Consultant, KAMC, Saudi Arabia, for statistical revision of the study.

\section{Funding: None.}

Competing interests: None declared.

\section{References}

1. The International Agency for Research on Cancer. International Childhood Cancer Day: much remains to be done to fight childhood cancer. 15 February 2016 (http://www.iarc.fr/ en/media-centre/pr/2016/pdfs/pr241_E.pdf, accessed on 23 March 2016).
2. Stefan DC, Siemonsma F. Delay and causes of delay in the diagnosis of childhood cancer in Africa. Pediatr Blood Cancer. 2011 Jan;56(1):80-5. PMID:21108442

3. Bonilla M, Moreno N, Marina N, deReyes G, Shurtleff SA, Downing JR, et al. Acute lymphoblastic leukemia in a devel- 
oping country: preliminary results of a nonrandomized clinical trial in El Salvador. J Pediatr Hematol Oncol. 2000 Nov-Dec;22(6):495-501. PMID:11132215

4. Howard SC. Wilimas JA. Delays in diagnosis and treatment of childhood cancer: where in the world are they important? Pediatr Blood Cancer. 2005 Apr;44(4):303-4. PMID:15486952

5. Dang-Tan T, Franco EL. Diagnosis delays in childhood cancer: a review. Cancer. 2007 Aug 15;110(4):703-13. PMID:17620277

6. Abdelkhalek E, Sherief L, Kamal N, Soliman R. Factors associated with delayed cancer diagnosis in egyptian children. Clin Med Insights Pediatr. 2014 Sep 4;8:39-44. PMID:25232287

7. Dang-Tan T, Trottier H, Mery LS, Morrison HI, Barr RD, Greenberg $\mathrm{ML}$, et al. Delays in diagnosis and treatment among children and adolescents with cancer in Canada. Pediatr Blood Cancer. 2008 Oct;51(4):468-74. PMID:18454472

8. Araz NC, Guler E. Delays in diagnosis of childhood cancer in southeastern Turkey and the associated factors. Pediatr Hematol Oncol. 2015 Mar;32(2):153-63. PMID:24498898

9. Halperin EC, Watson DM, George SL. Duration of symptoms prior to diagnosis is related inversely to presenting disease stage in children with medulloblastoma. Cancer. $2001 \mathrm{Apr}$ 15;91(8):1444-50. PMID:11301391

10. Crawford JR, Zaninovic A, Santi M, Rushing EJ, Olsen $\mathrm{CH}_{\text {, }}$ Keating RF, et al. Primary spinal cord tumors of childhood: effects of clinical presentation, radiographic features, and pathology on survival. J Neurooncol. 2009 Nov;95(2):259-69. PMID:19521666

11. Widhe $B$, Widhe T. Initial symptoms and clinical features in osteosarcoma and Ewing sarcoma. J Bone Joint Surg Am. 2000 May;82(5):667-74. PMID:10819277

12. Brasme JF, Morfouace M, Grill J, Martinot A, Amalberti R, Bons-Letouzey $\mathrm{C}$, et al. Delays in diagnosis of paediatric cancers: a systematic review and comparison with expert testimony in lawsuits. Lancet Oncol. 2012 Oct;13(10):e445-59. PMID:23026830

13. Loh AH, Aung L, Ha C, Tan AM, Quah TC, Chui CH. Diagnostic delay in pediatric solid tumors: a population based study on determinants and impact on outcomes. Pediatr Blood Cancer. 2012 Apr;58(4):561-5. PMID:22052842

14. Haimi M, Peretz Nahum M, Ben Arush MW. Delay in diagnosis of children with cancer: a retrospective study of 315 children. Pediatr Hematol Oncol. 2004 Jan-Feb;21(1):37-48. PMID:14660305

15. Haimi M, Perez-Nahum M, Stein N, Ben Arush MW. The role of the doctor and the medical system in the diagnostic delay in pediatric malignancies. Cancer Epidemiol. 2011 Feb;35(1):83-9. PMID:20685192 